\title{
Simple and Accurate Two-Dimensional Angle Estimation for a Single Source With Uniform Circular Array
}

\author{
Yuntao Wu and H. C. So, Senior Member, IEEE
}

\begin{abstract}
A least squares algorithm for two-dimensional (2-D) angle estimation of a single narrowband source impinging on a uniform circular array (UCA) is presented. Utilizing the centrosymmetric array configuration, a new correlation-based method is devised to obtain closed-form estimates of the azimuth and elevation angles. Although the proposed method is computationally simpler than the conventional schemes, computer simulations show that it can approach optimum estimation performance.
\end{abstract}

Index Terms-Array signal processing, circular array, two-dimensional (2-D) angle estimation.

\section{INTRODUCTION}

$\mathbf{E}$ STIMATING the two-dimensional (2-D) directions-of-arrival, namely, the azimuth and elevation angles, of propagating plane waves incident on a planar array is an important problem in array signal processing due to its applications in radar, sonar, mobile communications, and so on. In particular, uniform circular array (UCA) is extensively utilized in the context of 2-D angle estimation due to its attractive advantages, including $360^{\circ}$ azimuthal coverage, almost unchanged directional pattern and additional elevation angle information. Common methods for 2-D angle estimation with UCAs include 2-D multiple emitter location and signal parameter estimation (MUSIC) [1], UCA-real-beam-MUSIC [2], UCA-estimation of signal parameters via rotational invariance techniques (ESPRIT) [2], modified 2-D MUSIC [3], and global matched filter-based method [4]. Among them, the subspace-based methods [1]-[3] require eigenvalue decomposition and 2-D search while [4] needs only 2-D search. Although the phase mode excitation-based UCA-ESPRIT method [2] provides a closed-form solution for the azimuth and elevation angles in the case of a single source, it still necessitates to perform eigenvalue decomposition.

In this letter, a novel closed-form solution for 2-D angle estimation of a single narrowband source with a UCA is developed. Our main idea is to utilize the phase of an autocorrelation sequence constructed from the centrosymmetrical array outputs, from which a set of linear equations for solving the 2-D angle

Manuscript received November 15, 2007; revised December 22, 2007.

The authors are with the Department of Electronic Engineering, City University of Hong Kong Kowloon, Hong Kong (e-mail: hcso@ee.cityu.edu.hk).

Color versions of one or more of the figures in this letter are available online at http://ieeexplore.ieee.org.

Digital Object Identifier 10.1109/LAWP.2008.916687

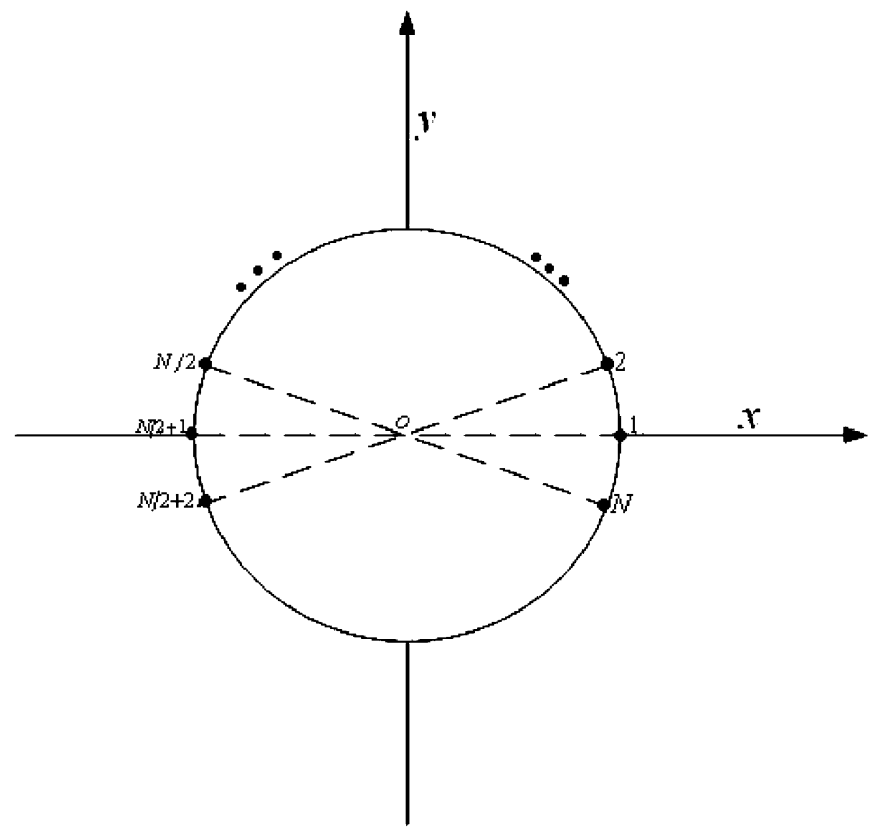

Fig. 1. Centrosymmetric circular array configuration.

pair can be obtained. As a result, the proposed algorithm is computationally simpler than the conventional schemes which requires 2-D search and/or eignenvalue decomposition. Nevertheless, its estimation performance can attain Cramér-Rao lower bound (CRLB) at sufficiently high signal-to-noise ratio (SNR) conditions.

\section{PROPOSED METHOD}

Consider a centrosymmetric circular array with $N$ omnidirectional sensors, where $N$ is even, and its configuration is shown in Fig. 1. Assume that a single narrowband source impinges on this UCA with radius $r$ from 2-D angle pair $(\theta, \phi)$ where $\theta$ and $\phi$ are the azimuth and elevation angles, respectively. The origin of the coordinate system is located at the center of the array.

The output of the $k$ th sensor of the circular array can be written as [2]

$$
\begin{aligned}
x_{k}(t) & =e^{j \frac{2 \pi r}{\lambda} \cos \left(\theta-\gamma_{k}\right) \sin (\phi)} s(t)+n_{k}(t) \\
k & =1,2 \cdots, N, \quad t=1,2 \ldots, K
\end{aligned}
$$

where $s(t)$ is the zero-mean and complex narrowband signal source with power $\sigma_{s}^{2}$, each of $\left\{n_{k}(t)\right\}$ is assumed to be a second-order ergodic, zero-mean, spatially and temporally 
white complex Gaussian process, which is independent of $s(t)$. Each sensor receives $K$ samples, $\lambda$ is the wavelength of the source and $\gamma_{k}=2 \pi(k-1) / N$ is the angle of the $k$ th sensor measured counterclockwise from the $x$ axis. The azimuth angle $\theta \in[0,2 \pi)$ of the signal is measured counterclockwise from the $x$ axis and the elevation angle $\phi \in[0, \pi)$ is measured down from the $z$ axis. Given $\left\{x_{k}(t)\right\}$, our task is to estimate $(\theta, \phi)$.

From the centrosymmetrical array configuration with even number of sensors, we have $\gamma_{N / 2+k}=\gamma_{k}+\pi$ and thus

$$
\begin{aligned}
a_{k}(\theta, \phi) & =a_{k+N / 2}^{*}(\theta, \phi) \\
k & =1,2, \ldots, N / 2
\end{aligned}
$$

where $a_{k}(\theta, \phi)$ denotes the complex array response of the signal at the $k$ th sensor and $*$ is the complex conjugation operator.

By utilizing (2), we first construct a sequence based on the autocorrelation of $\left\{x_{k}(t)\right\}$

$$
\begin{aligned}
p(k) & =\frac{1}{K} \sum_{t=1}^{K} x_{k}(t) x_{k+N / 2}^{*}(t) \\
k & =1,2 \cdots, N / 2 .
\end{aligned}
$$

When both $K$ and SNR are sufficiently large and assuming that ergodicity holds, $p(k)$ can be approximated as

$$
\begin{aligned}
p(k) & \approx \sigma_{s}^{2} a_{k}(\theta, \phi) a_{k+N / 2}^{*}(\theta, \phi) \\
& =\sigma_{s}^{2} e^{j \frac{4 \pi r}{\lambda} \cos \left(\theta-\gamma_{k}\right) \sin (\phi)} \\
k & =1,2 \cdots, N / 2 .
\end{aligned}
$$

Let $\omega_{k}$ be the phase angle of $p(k)$. From (4), we develop the following relationship:

$$
\begin{aligned}
& \omega_{k} \approx \frac{4 \pi r}{\lambda} \cos \left(\theta-\gamma_{k}\right) \sin (\phi)+2 \pi m_{k} \\
& k=1,2 \cdots, N / 2
\end{aligned}
$$

where $m_{k}$ is an integer. Note that the circular arrays are usually designed such that either $r / \lambda=0.5$ or contiguous elements are separated by less than $\lambda / 2$ [4]. For a UCA with six or more elements, we have $r / \lambda \geq 0.5$, that is, the separation of pairs of elements at the opposite ends will be at least $\lambda$. In this case, there will exist at least two arrival directions giving rise to the same cross covariance between a given set of receiver pairs, which leads to the phase ambiguity term of $2 \pi m_{k}$ in (5). Since the phase/angle ambiguity problem has been well addressed in the literature, the interested reader is referred to [5] and [6] for the solutions. In the following, we assume that the phase ambiguity has been successfully tackled and thus unambiguous 2-D angle estimation is achieved. With $m_{k}=0,(5)$ can be written as

$$
\begin{aligned}
\omega_{k} & \approx \frac{4 \pi r}{\lambda}\left[\begin{array}{ll}
\cos \left(\gamma_{k}\right) & \sin \left(\gamma_{k}\right)
\end{array}\right]\left[\begin{array}{c}
\sin (\phi) \cos (\theta) \\
\sin (\phi) \sin (\theta)
\end{array}\right] \\
k & =1,2 \cdots, N / 2 .
\end{aligned}
$$

Expressing (6) in matrix form yields

$$
\mathrm{Ab} \approx \mathrm{w}
$$

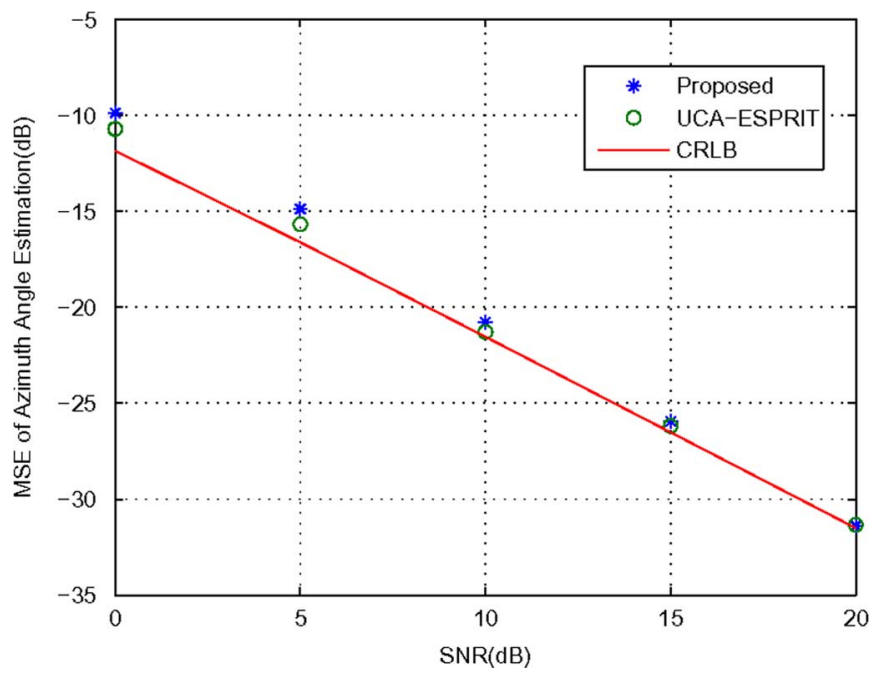

Fig. 2. MSE of azimuth angle versus SNR.

where

$$
\begin{aligned}
& \mathbf{A}=\left[\begin{array}{cc}
\cos \left(\gamma_{1}\right) & \sin \left(\gamma_{1}\right) \\
\vdots & \vdots \\
\cos \left(\gamma_{N / 2}\right) & \sin \left(\gamma_{N / 2}\right)
\end{array}\right] \\
& \mathbf{b}=\frac{4 \pi r}{\lambda}[\sin (\phi) \cos (\theta) \quad \sin (\phi) \sin (\theta)]^{T}
\end{aligned}
$$

and

$$
w=\left[\omega_{1}, \ldots, \omega_{N / 2}\right]^{T}
$$

with $T$ denotes the transpose operator.

The least squares (LS) estimate of $\mathbf{b}$, denoted by $\hat{\mathbf{b}}$, is

$$
\hat{\mathbf{b}}=\left[\begin{array}{ll}
\hat{b}_{1} & \hat{b}_{2}
\end{array}\right]^{T}=\left(\mathbf{A}^{T} \mathbf{A}\right)^{-1} \mathbf{A}^{T} \mathbf{w} .
$$

The estimate of the angle pair $(\theta, \phi)$, denoted by $(\hat{\theta}, \hat{\phi})$ is then determined from $\hat{b}$

$$
\hat{\theta}=\tan ^{-1}\left(\frac{\hat{b}_{2}}{\hat{b}_{1}}\right)
$$

and

$$
\hat{\phi}=\sin ^{-1}\left(\frac{\lambda}{4 \pi r} \sqrt{\hat{b}_{1}^{2}+\hat{b}_{2}^{2}}\right) .
$$

In the following, a simple procedure for unambiguous 2-D angle estimation for the case of $N=4$ is presented as this scenario corresponds to the $\pi$ ambiguity problem instead of the typical $2 \pi$ ambiguity problem. We utilize the signs of $\hat{b}_{1}$ or $\hat{b}_{2}$ to distinguish between the arrivals from $0^{\circ}, 90^{\circ}, 180^{\circ}$, and $270^{\circ}$ : If $\hat{b}_{1}=0$, then we get the correct estimation of $\theta$ by the sign of $\hat{b}_{2}$, that is, $\hat{\theta}=\pi-\operatorname{sgn}\left(\hat{b}_{2}\right) \pi / 2$ where sgn denotes the sign operator. While if $\hat{b}_{2}=0$, we employ the sign of $\hat{b}_{1}$ to unambiguously estimate $\theta$ as $\hat{\theta}=\left(1-\operatorname{sgn}\left(\hat{b}_{1}\right)\right) \pi / 2$.

Regarding major computational complexity, the number of multiplications for calculating $\mathbf{b}$ includes $N K / 2$ in the autocorrelation computation and $O\left(N^{2}\right)$ in the LS procedure. On the other hand, the UCA-ESPRIT technique for a single source [2] involves $N^{2} K$ for cross-covariance computation and $O\left(N^{3}\right)$ for 


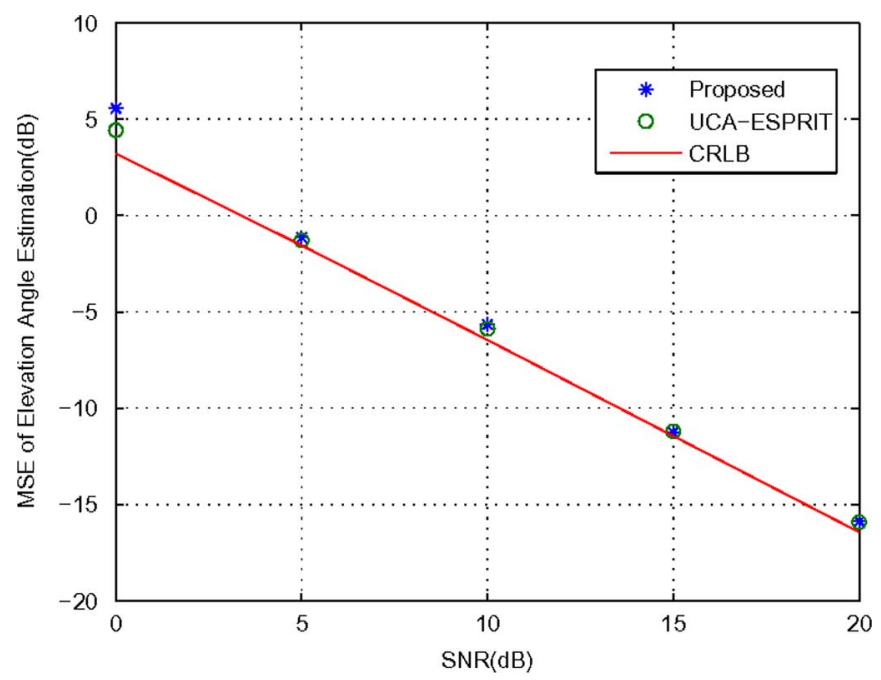

Fig. 3. MSE of elevation angle versus SNR.

the eigenvalue decomposition of the resultant covariance matrix. For the typical conditions of $K>N$, the ratio of the computational requirement of the proposed algorithm to that of [2] will be approximately $1 /(2 N)$, which indicates the computational attractiveness of our proposed approach.

\section{SimUlation ResUlts}

Computer simulations have been carried out to evaluate the performance of the proposed method for 2-D angle estimation of a single narrowband source in the presence of additive white Gaussian noise. We consider a UCA consisting of $N=12$ antennas with $r=\lambda$ and the signal impinges on the array from angle pair $\left(\theta=70^{\circ}, \phi=10^{\circ}\right)$. The number of samples is set to $K=100$ at each sensor. We employ the mean square error
(MSE) as the performance measure and all results provided are averages of 100 independent runs.

Figs. 2 and 3 show the MSEs for azimuth and elevation angles, respectively, versus different SNR conditions. The results using the UCA-ESPRIT algorithm [2] and CRLB are included to contrast the performance of the proposed algorithm. It is seen that its estimation accuracy is comparable to that of UCA-ESPRIT method and also close to the CRLB particularly at higher SNRs.

\section{CONCLUSION}

A simple and closed-form solution for 2-D angle estimation of a single narrowband source with a UCA has been devised. The key idea in the algorithm development is to utilize the phase of an autocorrelation sequence constructed from the centrosymmetrical array outputs. It is shown that the estimation performance of the proposed algorithm is comparable to that of the UCA-ESPRIT method [2] and attains CRLB at sufficiently high SNR conditions.

\section{REFERENCES}

[1] R. O. Schmidt, "Multiple emitter location and signal parameter estimation," IEEE Trans. Antennas Propag., vol. AP-34, no. 3, pp. 271-280, Mar. 1986.

[2] C. P. Mathews and M. D. Zoltowski, "Eigenstructure techniques for 2-D angle estimation with uniform circular arrays," IEEE Trans. Signal Process., vol. 42, no. 9, pp. 2395-2407, Sep. 1994.

[3] Z. Ye, L. Xiang, and X. Xu, "DOA estimation with circular array via spatial averaging algorithm," IEEE Antennas Wireless Propag. Lett., vol. 6, pp. 74-76, 2007.

[4] J.-J. Fuchs, "On the application of the global matched filter to DOA estimation with uniform circular arrays," IEEE Trans. Signal Process., vol. 49, no. 4, pp. 702-709, Apr. 2001.

[5] M. D. Zoltowski and C. P. Mathews, "Real-time frequency and 2-D angle estimation with sub-Nyquist spatio-temporal sampling," IEEE Trans. Signal Process., vol. 42, no. 10, pp. 2781-2794, Oct. 1994.

[6] K. R. Sundaram, R. K. Mallik, and U. M. S. Murthy, "Modulo conversion method for estimating the direction of arrival," IEEE Trans. Aerosp. Electron. Syst., vol. 36, no. 4, pp. 1391-1396, 2000. 